\title{
PEGylated Curcumin with Gold Nanoparticles: Antimicrobial Agent Evaluation
}

\author{
Courrol Daniella dos Santos ${ }^{1}$, Teixeira Bruna Henrique ${ }^{2}$, Pereira Camila Bueno Pacheco ${ }^{1}$, Franzolin \\ Marcia Regina $^{1}$, Courrol Lilia Coronato ${ }^{2}$ \\ ${ }^{1}$ Instituto Butantã, Laboratório de Bacteriologia, São Paulo, SP, Brazil. \\ ${ }^{2}$ Universidade Federal de São Paulo, Laboratório de Lasers e Óptica Biomédica Aplicada, Departamento de Ciências \\ Exatas e da Terra, Diadema, SP - Brazil \\ lccourrol@gmail.com
}

\section{Extended Abstract}

Curcumin is a polyphenol derived from the Curcuma Longa plant. Curcumin exhibits a variety of therapeutic properties, including antioxidant, analgesic, anti-inflammatory, anticancer and antiseptic activities [1].

Curcumin acts as photosensitizer in photodynamic therapy (PDT), which is an alternative way to kill pathogenic microorganisms [2]. The use of gold nanoparticles (AuNPs) as the vehicle for curcumin delivery increases photodynamic ability [3]. Therefore, the present study aim is to investigate the effect of the photodynamic action of LED-activated curcumin gold nanoparticles, on cell viability of Staphylococcus aureus, Staphylococcus aureus (Sa), Staphylococcus epidermidis (Se), Pseudomonas aeruginosa ( $\mathrm{Pa})$, Escherichia coli $(\mathrm{Ec})$, Citrobacter freundii $(\mathrm{Cf})$ and Klebsiella pneumoniae $(\mathrm{Kp})$.

To prepare Curcumin gold nanoparticles (Curc:AuNps) solutions, $3.2 \mathrm{mg}$ of $\mathrm{HAuCl}_{4}$ was mixed with $1.5 \mathrm{mg}$ Curcumin and Polyethylene glycol (PEG) in Mili-Q water. This solution was exposed to light from a mercury metal halide lamp. The resulting solutions were characterized by UV-Vis and transmission electron microscopy (TEM). The in vitro antibacterial activity of Curc:AuNps was evaluated against $S a, S e, P a, E c, C f$ and $K p$ using the minimum inhibitory concentration (MIC) test. The effects of exposure to yellow LED light with $\sim 38 \mathrm{~J} / \mathrm{cm}^{2}$ fluence and variations in the Curc:AuNPs concentrations (50, 75 and 100\% dilutions) were studied.

The presence of a surface plasmon resonance band at $\sim 520 \mathrm{~nm}$ indicated the formation of spherical gold nanoparticles. In the presence of PEG, the solution color changed some minutes after the mixing of reagents, and solution size homogeneity was improved with the mercury metal halide lamp illumination. TEM analyses showed $\sim 17 \pm 2 \mathrm{~nm}$ nanoparticles.

The results indicate that PDT with curcumin gold nanoparticles strongly inhibits the development of Gram positive and Gram negative bacteria. This study revealed antibacterial probability of inhibition $>80 \%$ with $32 \mathrm{mM}$ of Curc:AuNPs and light against $S a, S e, C f$ and $K p$ strains after 18 hours of incubation. NOVA/Tukey's tests were conducted to compare groups curcumin/Curc:AuNPs, with and without photodynamic excitation.

This work was supported by the "Fundação de Amparo à Pesquisa do Estado de São Paulo" (FAPESP), Grant number 2014/06960-9.

\section{References}

[1] S. C. Gupta, S. Patchva, and B. B. Aggarwal, "Therapeutic Roles of Curcumin: Lessons Learned from Clinical Trials," Aaps Journal, vol. 15, no. 1, pp. 195-218, 2013.

[2] V. Perez-Laguna, L. Perez-Artiaga, V. Lampaya et al., "In vitro effect photodynamic therapy with methylene blue, rose bengal, protoporphyrin IX and curcumin on Candida albicans, C. parapsilosis and C. krusei," Mycoses, vol. 58, pp. 204204, 2015.

[3] R. K. Gangwar, V. A. Dhumale, D. Kumari et al., "Conjugation of curcumin with PVP capped gold nanoparticles for improving bioavailability," Materials Science \& Engineering C-Materials for Biological Applications, vol. 32, no. 8, pp. 2659-2663, 2012. 